\title{
Building a learning community through collaborative, online assessment preparation
}

\author{
Ellie Davison \\ University of Lincoln, UK
}

Keywords: learning community; peer assessment; foundation; belonging.

\section{The challenge:}

As part of the skills development aspect of an integrated foundation year, students would previously have developed their academic writing during face-to-face sessions. The marking rubric would have been presented, with students invited to discuss how successfully previous submissions had met the criteria, and draft submissions swapped for peer feedback during group tutorials. However, each year, some students were reluctant to participate, perhaps due to a lack of confidence in sharing their opinions and critiquing each other's work in person. Furthermore, due to lockdown restrictions, face-to-face sessions were not possible; thus, could the pandemic provide the environment to improve the approach?

Peacock and Cowan (2019) report that students who feel they are integral to the activity and success of the whole cohort develop a sense of belonging, with Thomas, Herbert and Teras (2014) noting the importance of embedding peer collaboration into assessment for the success of online provision. Therefore, the challenge was to facilitate a supportive online learning community to allow students to benefit from each other's insights, with accessibility and flexibility embedded to support the diverse cohort. Furthermore, as students' 'academic self-concept' (their ability to visualise themselves succeeding in academia) (Brunner et al., 2010) is arguably as important as the development of subject knowledge for foundation cohorts, could academic confidence be built from afar? 


\section{The response:}

A suite of fully captioned, asynchronous micro-lectures, introducing the assessment brief, the marking rubric, and fundamentals of academic writing and referencing were produced to ensure that students could engage with this crucial content at a time that was convenient to them (e.g., fitting around caring or home schooling commitments). Students reported that they found the ability to re-watch videos at appropriate points in their assessment preparation hugely beneficial.

To facilitate interactivity, synchronous online sessions were delivered, using Talis Elevate to conduct group annotations of previous submissions. Students highlighted sections of the text and added comment boxes to identify areas they felt met aspects of the marking rubric and areas for improvement. Comments were threaded and nested to allow for dialogue and debate amongst the cohort and students had the option of commenting anonymously, encouraging less confident participants to engage. While interaction between students and familiarisation with the marking rubric were intended outcomes, an unexpected benefit was the lecturer's ability to add prompts in real time, direct the discussion, give positive encouragement, and clarify any areas of uncertainty. The activity also proved extremely insightful as to the efficacy of the preparatory teaching, showing which aspects of the task students had got to grips with and which areas of the provision might benefit from improvements. To allow for asynchronous contributions, as well as a later review of the discussion points, the annotated texts continued to be available until the end of the module.

To further cultivate a collaborative student support network, the evaluation skills developed through the group evaluation activity were then applied to online peer-feedback, as peer review has been demonstrated to simultaneously promote active engagement and necessitate not only interaction but dependence upon each other for overall success (Simonsmeier et al., 2020). Turnitin PeerMark was used to randomly allocate draft submissions amongst the cohort and the feedback was scaffolded through a series of question boxes, all aligned to the marking rubric, with each question requiring a minimum word response. The opportunity for lecturers to view all the peer feedback behind the scenes allowed any misconceptions to be corrected. Engagement was significantly higher with this online strategy than in previous face-to-face years and the quality of the feedback 
was rich, with the detailed suggestions as to how improvements could be made, instead of bland coverall statements, being a particular highlight.

\section{Recommendations:}

The described approach allowed students to apply the marking rubric to a variety of submissions prior to the final assessment deadline, generating internal feedback on their own submissions (Nicol, 2020), and resulting in excellent attainment despite the challenges of lockdown. Students valued the flexibility of both how and when to participate (anonymous vs. onymous; synchronous vs. asynchronous) and evaluating submissions of a range of quality was also effective; seeing common errors encouraged students not to make the same mistakes, while excellent examples stimulated students to improve their work accordingly. The random distribution of submissions for peer feedback also broadened the experience of students who otherwise may only have interacted with texts within their own subject area or tutor group.

The ability to provide high quality, constructive feedback is a key graduate skill (Nicol, Thomson and Breslin, 2014), but students need to learn how to do so effectively. In the peer review task instructions, a variety of examples of constructive feedback, specifically linked to how a suggested improvement might move a submission into the next marking bracket, were provided. The opportunity for students to practise this skill in the lecturer supported group evaluation was also crucial to the success of the peer review.

With a return to face-to-face sessions, this approach could facilitate truly blended sessions, synthesising verbal discussions with comments, using Talis, displayed on the lecture screen. For large cohorts, it may be useful to run parallel group annotations, perhaps with different submissions uploaded, to prevent the dialogue boxes from becoming too busy; students would then be able to access all evaluations following the session. Encouraging students to utilise their feedback can be a challenge. To address this, it could be that part of the assessment mark reflects how the peer reviews received have been used to improve the submitted work. Marks could also be awarded for the quality of the peer review itself. 
Analysis by Senior et al. (2018) revealed three major factors in fostering successful student engagement: opportunities to present opinions, an academic benefit of participation, and the development of professional skills. Scaffolded peer evaluation met these criteria to produce a vibrant online community and the approaches introduced will be maintained long after lockdown has lifted.

\section{References}

Brunner, M., Keller, U., Dierendonck, C., Reichert, M., Ugen, S., Fischback, A. and Martin, R. (2010) 'The structure of academic self-concepts revisited: the nested Marsh/Shavelson model', Journal of Educational Psychology 102(4), pp.964-981. https://doi.org/10.1037/a0019644

Nicol, D., Thomson, A. and Breslin, C. (2014) 'Rethinking feedback practices in higher education: a peer review perspective', Assessment and Evaluation in Higher Education, 39(1), pp.102-122. https://doi.org/10.1080/02602938.2013.795518.

Nicol, D. (2020) 'The power of internal feedback: exploiting natural comparison processes', Assessment and Evaluation in Higher Education. https://doi.org/10.1080/02602938.2020.1823314.

Peacock, S and Cowan, J. (2019) 'Promoting a sense of belonging in online learning communities of inquiry in accredited courses', Online Learning, 23(2), pp.67-81. http://dx.doi.org/10.24059/olj.v23i2.1488.

Senior, R. M., Bartholomew, P., Soor, A., Shepperd, D., Bartholomew, N. and Senior, C. (2018) 'The rules of engagement: student engagement and motivation to improve the quality of undergraduate learning', Frontiers in Education, 3. https://doi.org/10.3389/feduc.2018.00032.

Simonsmeier, B. A., Peiffer, H., Flaig, M. and Schneider, M. (2020) 'Peer feedback improves students' academic self-concept in higher education', Research in Higher Education, 61(6), pp.706-724. https://doi.org/10.1007/s11162-020-09591-y. 
Thomas, L., Herbert, J. and Teras, M. (2014) 'A sense of belonging to enhance participation, success and retention in online programs', The International Journal of the First Year in Higher Education, 5(2), pp.69-80.

https://doi.org/10.5204/intjfyhe.v5i2.233.

\section{Author Details}

Ellie Davison is the Director of Teaching and Learning for the Foundation Studies Centre, and a Director of Digital Engagement for the College of Science, at the University of Lincoln. 